\title{
Beneficial and Detrimental Remodeling of Glial Connexin and Pannexin Functions in Rodent Models of Nervous System Diseases
}

\author{
Lucila Brocardo, Luis Ernesto Acosta, Ana Paula Piantanida ${ }^{\dagger}$ and Lorena Rela* \\ Grupo de Neurociencia de Sistemas, Facultad de Medicina, Instituto de Fisiología y Biofísica Bernardo Houssay (IFIBIO \\ Houssay), Consejo Nacional de Investigaciones Cientificas y Técnicas (CONICET), Universidad de Buenos Aires, \\ Buenos Aires, Argentina
}

OPEN ACCESS

Edited by:

Giovanni Cirillo,

Università degli Studi della Campania

Luigi Vanvitelli, Italy

Reviewed by:

Parisa Gazerani,

Aalborg University, Denmark

Kenji Fabricio Shoji,

IRSET, France

*Correspondence:

Lorena Rela

lorena.rela@fmed.uba.ar

tPresent address:

Ana Paula Piantanida

Instituto de Virología, CICVyA,

Instituto Nacional de Tecnología Agropecuaria (INTA), Buenos Aires,

Argentina

Received: 31 July 2019 Accepted: 17 October 2019

Published: 06 November 2019

Citation:

Brocardo L, Acosta LE,

Piantanida AP and Rela L (2019) Beneficial and Detrimental Remodeling of Glial Connexin and

Pannexin Functions in Rodent

Models of Nervous System Diseases.

Front. Cell. Neurosci. 13:491.

doi: 10.3389/fncel.2019.00491
A variety of glial cell functions are supported by connexin and pannexin proteins. These functions include the modulation of synaptic gain, the control of excitability through regulation of the ion and neurotransmitter composition of the extracellular milieu and the promotion of neuronal survival. Connexins and pannexins support these functions through diverse molecular mechanisms, including channel and non-channel functions. The former comprise the formation of gap junction-mediated networks supported by connexin intercellular channels and the formation of pore-like membrane structures or hemichannels formed by both connexins and pannexins. Non-channel functions involve adhesion properties and the participation in signaling intracellular cascades. Pathological conditions of the nervous system such as ischemia, neurodegeneration, pathogen infection, trauma and tumors are characterized by distinctive remodeling of connexin expression and function. However, whether these changes can be interpreted as part of the pathogenesis, or as beneficial compensatory effects, remains under debate. Here we review the available evidence addressing this matter with a special emphasis in mouse models with selective manipulation of glial connexin and pannexin proteins in vivo. We postulate that the beneficial vs. detrimental effects of glial connexin remodeling in pathological conditions depend on the impact of remodeling on the different connexin and pannexin channel and non-channel functions, on the characteristics of the inflammatory environment and on the type of interaction among glial cells types.

Keywords: connexins, pannexins, gap junctions, hemichannels, astrocytes, microglia, plasticity

\section{INTRODUCTION}

Connexins are transmembrane proteins with a variety of physiological roles including channel and non-channel functions. Channel functions involve the formation of gap junctions, which are intercellular connections, permeable to ions and small metabolites, formed by the apposition of connexin hexamers (connexons) provided by each of the participating cells. 
Gap junction-mediated glial networks are ubiquitous in the central nervous system and involve astrocytes and oligodendrocytes. The type of connexins involved in the interaction depends on the cell types participating in the connection (Rouach et al., 2002; Giaume and Theis, 2010; Takeuchi and Suzumura, 2014; Abudara et al., 2018). Connexins can also function as membrane hemichannels with the potential to release mediators such as ATP and glutamate from astrocytes and microglia (Orellana et al., 2013; Gajardo-Gómez et al., 2016; Nielsen et al., 2017). Non-channel functions of connexins involve adhesion properties and intracellular cascade signaling (Zhou and Jiang, 2014; Leithe et al., 2018).

The ubiquitous nature and diversity of connexin functional properties make them versatile mediators of glial cell physiology and a putative locus for maladaptive plasticity in pathological conditions. Plastic changes in abundance and/or functions of glial connexins have been reported in a variety of pathology models (Belousov et al., 2017; De Bock et al., 2017; Xing et al., 2019). However, whether connexin plasticity is causal to the pathology, or is an epiphenomenon of pathological conditions, is becoming to be elucidated only recently, owing to the availability of tools for selective manipulation of glial connexins in vivo (Giaume and Theis, 2010). Here we review the available work using rodent models with glia-selective connexin manipulations to evaluate causal relations between glial connexin plasticity and neuropathology, focusing on traumatic and neurodegenerative diseases, ischemia and brain tumors. Most of the cases reviewed here will focus on connexin 43 ( $\mathrm{Cx} 43$ ), which is by far the most studied glial connexin and dominates the available literature. We will also review the scarce literature available on glia-selective pannexin manipulations in these models, and discuss the glia-selective data in the context of additionally more abundant data using global connexin and pannexin manipulations.

Modulation of connexin expression and function that may emerge in the context of hypoxia, inflammation and injury, both in vitro and in vivo, have been thoroughly reviewed and putative mechanisms underlying the observed changes have been proposed (Rouach et al., 2002; Contreras et al., 2004; Farahani et al., 2005; Kielian, 2008; Orellana et al., 2009; Eugenin et al., 2012; Koulakoff et al., 2012; Quintanilla et al., 2012; Bosch and Kielian, 2014; Freitas-Andrade and Naus, 2016). A few important points emerging from the body of available literature are worth mentioning before we focus on glia-selective in vivo manipulations, which is the focus of this review. The first point is that a change in connexin immunolabeling needs to be interpreted with caution. Connexin channels in the context of gap junction plaques or in a disassembled state can have dramatically different immunoreactive properties, thus connexins may appear absent owing to epitope masking (Theriault et al., 1997). This highlights the importance of using independent approaches to measure connexin abundance and function. A second point is that changes in the expression of glial connexins in pathological conditions are connexin-, time-, region- and modelspecific. As an example, Borna virus infection of neonatal rats results in neurodegeneration and widespread reactivity of hippocampal astrocytes when analyzed 2 months after infection (Köster-Patzlaff et al., 2007). This astrocytic activation is accompanied by an increase of $\mathrm{Cx} 43$ in all layers of the dentate gyrus of the hippocampus and a decrease of the same connexin in the CA3 pyramidal layer. Another typically astrocytic connexin, connexin $30(\mathrm{Cx} 30)$, increased in all the hippocampal regions mentioned above (Köster-Patzlaff et al., 2007). In contrast, in a mouse model proposed to mimic features of aging and Alzheimer's disease induced by ovariectomy and chronic D-galactose administration, a reduction of $\mathrm{Cx} 43$ in the dentate gyrus and CA1 regions of the hippocampus was observed, while no change was observed in the CA3 hippocampal region (Liu et al., 2010). This was suggested to reflect differential resistance to neuronal damage of CA3 compared to CA1, as was observed in a model of ischemia in rats (Rami et al., 2001). Thus, generalizations about connexin plasticity across brain regions and pathologies should be cautious. A third issue is that changes in the abundance of glial connexin proteins in pathological conditions is challenging to interpret without parallel assessments of functional studies of connexin functions, such as the extent of gap junction coupling, the permeability of connexin hemichannels available at the membrane surface (Sáez et al., 2013) and the phosphorylation state of specific connexin sites that can mediate intracellular signaling. These functions may be oppositely modulated and/or have opposing effects on the severity of the pathology (Retamal et al., 2007; Karpuk et al., 2011). Therefore, when manipulations target more than one connexin function-e.g., both gap junctions and hemichannels - this has to be clearly stated and the effects of the manipulation should be interpreted with care. Finally, an important idea, emerging mostly from in vitro studies is that different connexins are expressed in different cell types of the nervous system-i.e., neurons, astrocytes, oligodendrocytes and microglia-and may interact in complex ways when modulated in pathologic conditions. Even more, glial pannexin proteins, which form membrane channels showing structure and function similarity to connexin hemichannels, can also be modulated in inflammatory conditions and have been proposed to play a role in pathologic conditions (Freitas-Andrade and Naus, 2016; Lapato and Tiwari-Woodruff, 2018). The contribution of $\mathrm{Cx} 43$ and pannexin-1 (Px1) to hemichannel activity in astrocytes has been a matter of debate as different studies assigned a preponderant role to one or the other, depending on the conditions analyzed (Iglesias et al., 2009; Orellana et al., 2011). Hence, cell type-selective manipulations of connexins and pannexins are essential to achieve a comprehensive idea of their roles in pathology. With these general ideas in mind, we will review the available literature that aims at using in vivo selective manipulations of glial connexin and pannexin functions to address their roles in a subset of pathologic conditions.

For this purpose, we used keyword search in PubMed and Scopus citation databases using combinations of keywords (Table 1). We then reviewed the methods section of all articles yielded by each search and selected those articles that used in vivo rodent models. A final selection of work using global and cell-specific connexin and pannexin manipulations were selected for detailed analysis. 
TABLE 1 | Number of original research articles found for the keyword combinations used for the literature search in PubMed and Scopus citation databases.

\begin{tabular}{lcc}
\hline & Connexin & Pannexin \\
\hline Astrocyte & 875 & 83 \\
Microglia & 150 & 39 \\
Oligodendrocyte & 212 & 5 \\
Ependymal & 28 & 1 \\
Endothelial + Brain & 93 & 6 \\
Tumor + Brain & 212 & 19 \\
\hline
\end{tabular}

\section{NEURODEGENERATIVE DISEASES}

We mentioned that connexins are expressed in different glial types and may interact in complex ways. To illustrate these complex interactions, we can mention a study by Orellana et al. (2011) that addressed the involvement of glial connexins and pannexins in neuronal death associated with exposure to a toxic fragment of the amyloid precursor protein, whose accumulation is associated with Alzheimer's disease. Orellana et al. (2011) observed that in cultured cortical astrocytes and microglia, toxic amyloid fragments increased hemichannel activity. While astrocyte hemichannel activity was explained mainly by $\mathrm{Cx} 43$ availability, microglial hemichannel activity involved both $\mathrm{Cx} 43$ and Px1 (Orellana et al., 2011). This amyloidinduced glial hemichannel activity was associated with the release of metabolites that are toxic for neurons, likely ATP and glutamate. While microglia-mediated neurotoxicity could be prevented by combined application of Px1 and Cx43 blockers, astrocyte-mediated neurotoxicity was abolished by $\mathrm{Cx} 43$ block alone (Orellana et al., 2011). In this same study, hippocampal slices in organotypic culture exposed to the toxic amyloid fragment showed increased hemichannel activity. This effect was revealed by dye uptake first in microglia, then in astrocytes and finally in neurons. While $\mathrm{Cx} 43$ blockers and microglia inactivation with minocycline completely prevented amyloidinduced neuronal death, in hippocampal slices obtained from mice with astrocyte-selective deficiency of $\mathrm{Cx} 43$ there was only a partial protection (Orellana et al., 2011). These data suggest that astrocytic recruitment is downstream microglial activation, however, pure astrocyte cultures can produce neurotoxic conditioned medium in the presence of toxic amyloid fragments. The relative contributions of microglia and astrocyte connexins to amyloid-induced neurotoxicity will need microglia-selective manipulations (Parkhurst et al., 2013; Yona et al., 2013; Zhao et al., 2019) of connexin and/or pannexin proteins for further clarification.

It is important to note that amyloid neurotoxicity modeled in cell and organotypic slice culture systems involve a rather homogeneous exposure to the toxic fragment. This homogeneous exposure does not reproduce the neurotoxicity gradients generated in the pathology characterized by amyloid deposition in the form of amyloid plaques. The relevance of these gradients for glial plasticity in Alzheimer's disease was nicely illustrated in a mouse model of familial Alzheimer's disease $\left(\mathrm{APP}_{\text {swe }} / \mathrm{PS}_{\mathrm{dEg}}\right.$ mice, or APP/PS1 mice) characterized by aging-dependent plaque formation. Hemichannel activity assessed using dye uptake in acute brain slices was enhanced in hippocampal astrocytes of APP/PS1 mice when compared to control mice. Interestingly, hemichannel hyperactivity in APP/PS1 astrocytes involved Cx43 and Px1 when astrocytes were located near amyloid plaques, while the increased hemichannel activity observed in astrocytes far from plaques involved only Cx43 (Yi et al., 2016). Microglial inhibition by minocycline partially reduced hemichannel hyperactivity in astrocytes near plaques, while astrocytes far from plaques remained unaffected. When the effects of the APP/PS1 mutations on hemichannel activity were analyzed in the context of a selective deficiency of $\mathrm{Cx} 43$ in astrocytes, almost no hemichannel activity was observed in astrocytes far from plaques, but a Px1 component of hemichannel activity remained in astrocytes near plaques. Thus, Px1 appears to contribute to astrocyte hemichannel activity in some in vivo conditions (Yi et al., 2016). Together with the prevention of hemichannel activity in astrocytes, the astrocyte-selective $\mathrm{Cx} 43$ deficiency in APP/PS1 mice was associated with less mitochondrial oxidative stress assessed by MitoSOX superoxide indicator and less dystrophic dendrites, assessed by reticulon 3 (RTN3) immunoreactivity (Yi et al., 2016), indicating that astrocyte $\mathrm{Cx} 43$ worsened the neurotoxicity of the environment. Of interest, no differences were observed in indicators of astrocytic gap junction coupling between APP/PS1 and control mice, assessed by fluorescence recovery after photobleaching (FRAP; Yi et al., 2016), strengthening the idea that the neurotoxic effect of astrocytes was exerted via $\mathrm{Cx} 43$ hemichannels. Further, APP/PS1 mice with astrocyte Cx43 deficiency had dramatically reduced astrogliosis and improved cognitive performance compared with APP/PS1 mice. Even more, re-expression of $\mathrm{Cx} 43$ in $\mathrm{Cx} 43$-deficient APP/PS1 mice using an astrocyte-directed adeno-associated viral vector reinstated the cognitive impairment (Ren et al., 2018). These results indicate that in the sequence of events of microglial and astrocytic activation that were proposed to lead to neuronal damage in Alzheimer's disease based on in vitro studies, the increase of $\mathrm{Cx} 43$ hemichannel activity in astrocytes is a deleterious process and this is confirmed in an in vivo model that selectively manipulates connexins in astrocytes.

Evidence for the idea that connexins participate in the complex interaction between astrocytes and microglia during neuroinflammation was provided as well by studies done in experimental autoimmune encephalomyelitis, a mouse model of multiple sclerosis. In this model, the pathologic condition is produced by subcutaneous immunization with myelin oligodendrocyte glycoprotein $\mathrm{MOG}_{35-55}$ peptide and intraperitoneal administration of Pertussis toxin on the day of immunization and 2 days after (Chen and Brosnan, 2006). This model is characterized by progressive paralysis followed by partial remission after an acute peak of clinical signs, associated with inflammatory infiltrate, demyelination and axonal damage (Chen and Brosnan, 2006). Mice with a selective astrocytic $\mathrm{Cx} 43$ deficiency or with this deficiency in a global Cx30 knockout background to prevent compensatory effects were not statistically different from wild type mice in terms of a clinical score that 
evaluates the severity and time course of the motor signs of experimental autoimmune encephalomyelitis up to 3-4 weeks after immunization (Lutz et al., 2012). These mice were not different either in terms of the degree of immune cell reactivity in the spinal cord, evaluated using ionized calcium binding adaptor molecule 1 (Iba1) immunostaining at the endpoint of clinical evaluation. Interestingly, the global Cx30 deficiency reduced the severity of the chronic phase ( 8 weeks after the insult) in this model and this effect was correlated with the emergence of an anti-inflammatory phenotype in both spinal cord astrocytes and microglia (Fang et al., 2018). Given that a reduction in the abundance of $\mathrm{Cx} 43$ during the chronic phase of experimental autoimmune encephalomyelitis was observed in global Cx30 deficient mice, the neuroprotective effect could be mediated indirectly via astrocytic Cx43. This possibility will need to be specifically tested ideally using conditional astrocytic-selective and inducible $\mathrm{Cx} 43$ deficiency alone or in combination with $\mathrm{Cx} 30$ deficiency. Furthermore, global Px1 deficiency delayed the onset of symptoms in this model of experimental autoimmune encephalomyelitis, improving the clinical state of mice in the acute phase. However, Px1-deficient mice reached a clinical score that was comparable to the one observed in wild type mice during the chronic phase (Lutz et al., 2013). When ATP release was evaluated in the bathing medium of spinal cord slices, the authors observed that the enhancement of ATP release that the insult produced was significantly smaller in slices obtained from Px1-deficient mice when compared to wild type slices (Lutz et al., 2013). The former results are compatible with a sequential involvement of Px1 and $\mathrm{C} \times 30$-and possibly $\mathrm{C} \times 43$ - in the progression of experimental autoimmune encephalomyelitis. Further experiments using glial cell type-selective pannexin and connexin manipulations will help to determine whether an early microglia dependent process involving Px1 elicits a late astrocyte-dependent process involving connexins as part of the pathogenesis of experimental autoimmune encephalomyelitis.

The importance of connexin and pannexin functions to explain pathological conditions characterized by neuronal dysfunction and degeneration is highlighted by data coming from studies of human diseases linked to connexin and pannexin mutations. X-linked Charcot-Marie-Tooth disease type 1 (OMIM \#302800) is characterized by progressive muscle weakness and atrophy associated with mutations in the GJB1 gene, coding for connexin $\mathrm{Cx} 32$ ( $\mathrm{Cx} 32)$. These mutations are linked to peripheral demyelination and axonal degeneration that can be accompanied by central manifestations (Wang and Yin, 2016). Deletions of the GJB1 coding sequence in humans produce a predominantly peripheral phenotype that is compatible with the alterations observed in Cx32 null mice (Hahn et al., 2000; Nakagawa et al., 2001). The central manifestations can be transient, do not appear to correlate with the severity of peripheral neuropathy and may be triggered by a variety of stress stimuli (Wang and Yin, 2016). Besides peripheral signs of neuropathy, Cx32 knockout mice show increased signs of widespread central nervous system inflammation when compared to wild type mice, both in basal conditions and after a systemic challenge with lipopolysaccharide (Olympiou et al., 2016). Interestingly, Cx32 knockout mice bearing a human Cx32 mutation associated with central manifestations of X-linked Charcot-Marie-Tooth disease (Cx32KOT55I) display an enhanced widespread central nervous system inflammatory response not associated with oligodendrocyte loss after lipopolysaccharide challenge, when compared with Cx32 knockout mice that do not carry this mutation (Olympiou et al., 2016). These data suggest that $\mathrm{C} \times 32$ mutations contribute to the disease not only through loss-of-function mechanisms that promote central neuroinflammation in addition to peripheral neuropathy, but also through gain-of-function mechanisms that increase the susceptibility to inflammatory challenges. The signals underlying the enhanced neuroinflammation remain to be elucidated.

Another demyelinating disease associated with mutations in the GJC2 gene coding for connexin $47(\mathrm{Cx} 47)$ is PelizaeusMerzbacher-like disease type 1 (OMIM \#608804), characterized by progressive spasticity and ataxia. When compared with wild type mice, both knockout mice for $\mathrm{Cx} 47$ and mice carrying a mutation associated with the disease in humans (Cx47KOM282T) show delayed myelination and mild motor impairment during the juvenile stage, together with cerebellar astro- and microgliosis, that is no longer observable in the adult stage owing to compensation by Cx32 (Tress et al., 2011). As is the case for $\mathrm{Cx} 32$-deficient and mutant mice, $\mathrm{Cx} 47$-deficient and mutant mice show evidence of gap junction disassembly, not only in relation to oligodendrocyte-oligodendrocyte connections but also for oligodendrocyte-astrocyte connections (Tress et al., 2011; Olympiou et al., 2016). Whether augmented signs of neuroinflammation in these connexin-deficient mice is associated with oligodendrocytic, astrocytic or neuronal dysfunction, or with a combination of signals from these cell types, remains to be established.

\section{ISCHEMIA}

The question whether astrocytic connexins are beneficial or detrimental in pathological conditions fueled a rich debate and is well illustrated for the case of ischemia (Giaume et al., 2007). Correlational evidence and nonselective manipulations aiming at inhibition of $\mathrm{Cx} 43$ function in astrocytes led to the idea that $\mathrm{Cx} 43$ contributed to the amplification of ischemic damage, as the treatment with octanol, a nonselective connexin and gap junction blocker, reduced the infarct size in rat models of cerebral ischemia by carotid or middle cerebral artery occlusion (Rawanduzy et al., 1997; Rami et al., 2001).

Contrasting with the former data, in a mouse model of ischemia produced by middle cerebral artery occlusion during $45 \mathrm{~min}$ with evaluation $24 \mathrm{~h}$ later, the infarct size was larger in mice with a selective $\mathrm{Cx} 43$ astrocytic deficiency when compared to wild type mice (Lin et al., 2008). In addition, an experience of hypoxic preconditioning of $5 \mathrm{~h}$ at $8 \%$ oxygen 3 days prior to artery occlusion produced an approximate 50\% reduction in infarct size in wild type mice, and was absent in mice with astrocytic Cx43 deficiency (Lin et al., 2008). The protective effect of hypoxic preconditioning needed the 
availability of adenosine A1 receptors and it was proposed to be produced by ATP release by $\mathrm{Cx} 43$ hemichannels and subsequent metabolization to adenosine extracellularly (Lin et al., 2008). Given that astrocytic Cx43 deficiency is partly compensated by an increase in $\mathrm{Cx} 30$ expression, the mice used in these experiments were also global Cx30 knockouts (Wallraff et al., 2006). Cx43 was proposed as pivotal for neuroprotection in this cerebral ischemia model and was reinforced by consistent results obtained in mice with conditional deletion of $\mathrm{Cx} 43$ in astrocytes and no deletion of Cx30 (Nakase et al., 2003). In these mice, more cell death and enhanced number of inflammatory cells were observed in the area of penumbra without significant changes in the volume affected by astrogliosis, when compared with control mice (Nakase et al., 2004). The protective effect of $\mathrm{Cx} 43$ against ischemic damage was further reinforced by another study using a mouse line bearing a $\mathrm{Cx} 43$ mutation that affects $\mathrm{Cx} 43$-mediated gap junction and hemichannel activity (Gjal ${ }^{\mathrm{Irt}}$; Kozoriz et al., 2013). Thus, in contrast to what was observed for the inflammatory environment of Alzheimer's disease models, $\mathrm{Cx} 43$ was regarded as neuroprotective under hypoxic conditions. One important difference called into attention to explain the difference between these two conditions is the chronicity of the insult in Alzheimer's disease models compared to the acute insult in the models of ischemia.

Genetic models that allow researchers to restrict the connexin deficiency to specific glial types were a big step forward to address glial connexin functions in nervous system pathology; however, there are a series of confounding effects that the strategy does not address. First, most of the data come from constitutive lines that tie the connexin deletion of interest to the timing of expression of a particular promoter, such as the promoter for glial fibrillary acidic protein (GFAP), which is also expressed by neural progenitor cells (Giaume and Theis, 2010). This caveat can be minimized with new tools that spare neural progenitor cells (Tsai et al., 2012; García-Cáceres et al., 2016; Srinivasan et al., 2016; Koh et al., 2017) or inducible tools that allow the researchers to evaluate phenomena in time windows shorter than the time needed for generation of new neurons (Burns et al., 2007; Rivers et al., 2008). Second, the deletion of connexin coding sequences eliminates all connexin functions, thus, it cannot address hemichannel, gap junction and non-channel connexin functions separately. This is particularly relevant in light of data showing that the permeability of gap junction channels and hemichannels can be oppositely modulated in an in vitro model of injury in which hemichannels were activated and gap junction coupling was reduced (Retamal et al., 2007). A promising tool to dissociate $\mathrm{Cx} 43$ hemichannel and gap junction channel functions is the connexin mimetic peptide Gap19, which blocks Cx43 hemichannels within the hour after exposure without affecting Cx43-mediated gap junction coupling. At larger exposure times, it slightly enhances gap junction coupling, so it nicely dissociates the two functions (Wang et al., 2013). A recent study assessed the effects of this peptide in male mice subjected to a cerebral ischemia-reperfusion protocol (45 min of middle cerebral artery occlusion and assessment $24 \mathrm{~h}$ after initiation of reperfusion). Intracerebroventricular administration of Gap19
$30 \mathrm{~min}$ before ischemia had a protective effect, significantly reducing infarct volume (Chen et al., 2019). This result indicates that $\mathrm{Cx} 43$ hemichannel function is deleterious in ischemia and suggests that the increase in infarct volume observed in mice with astrocytic Cx43 deficiency (Nakase et al., 2004) obeys to a neuroprotective role of $\mathrm{Cx} 43$-mediated gap junction channels that overrides the neurotoxic action of hemichannels. However, as Gap19 also downregulated Cx43 expression by about $30 \%$ and astrocytic gap junction connectivity was not assessed (Chen et al., 2019), a contribution of astrocytic gap junctions to explain the neuroprotective effect of Gap19 cannot be ruled out. Potential effects of Gap19 in microglia or other targets could also explain the results, as the manipulation was not cell-type selective. Further support for the idea that an increment of astrocytic $\mathrm{Cx} 43$ hemichannel activity in response to ischemic insults is detrimental comes from a study using mice with global site-directed mutagenesis to eliminate functional phosphorylation sites that are a substrate for mitogen-activated protein kinase in the C-terminus of $\mathrm{Cx} 43$ (Freitas-Andrade et al., 2019). These mice (MK4 mice) show reduced infarct size after permanent middle cerebral artery occlusion when compared with wild type mice and two other mouse lines targeting phosphorylation sites for protein kinase $\mathrm{C}$ and caseine kinase 1, these two displaying no protection. Cultured astrocytes from MK4 mice showed reduced hemichannel activity assessed using dye uptake and electrophysiological measurements. In addition, MK4 astrocyte gap junction coupling assessed through a scrapeloading assay in cortical brain slices was indistinguishably increased in MK4 and wild type mice after the ischemic insult (Freitas-Andrade et al., 2019). Given that this was a global manipulation, it remains to be confirmed that the protective effect is astrocytic Cx43-dependent.

Non-channel functions of $\mathrm{Cx} 43$ involve adhesion properties and the participation in intracellular signaling via the C-terminal domain. $\mathrm{Cx} 43$ is codified by the Gjal gene, which can produce alternative carboxyl-terminal fragments via translation at alternative initiation sites (Ul-Hussain et al., 2014). Among these fragments, the GJA1-20k variant is selectively upregulated in a rat model of brain hypoxia-ischemia and may explain at least part of the neuroprotective effects produced by $\mathrm{Cx} 43$ in hypoxic conditions. Mice with a C-terminal-truncation in the Cx43 coding region show more vulnerability to brain ischemia, however, as astrocytic gap junction and hemichannel functions are partially disrupted as well in these mice, a neuroprotective role of the carboxyl-terminal fragment of $\mathrm{Cx} 43$, independent of its impact in channel functions, cannot be confirmed (Kozoriz et al., 2010).

Multiple connexins and glial types likely contribute to the outcome after ischemic damage. This idea is reinforced by the observation that male null mice for $\mathrm{Cx} 32$ are more vulnerable to global cerebral ischemia produced by transient $(10 \mathrm{~min})$ bilateral common carotid artery occlusion (Oguro et al., 2001). This enhanced vulnerability was observed 7 days after the insult as increased neuronal loss in the CA1 hippocampal region of null mice when compared with wild type mice (Oguro et al., 2001). Given that Cx32 is expressed by oligodendrocytes and by parvalbumin-positive inhibitory interneurons in the 
hippocampus, the locus of origin of the Cx32-dependent neuroprotective effect could not be elucidated in this model, highlighting the need of glia type-selective manipulations of connexin proteins to address their functions (Doerflinger et al., 2003; Piantanida et al., 2019).

The contribution of pannexin proteins to the outcome after ischemic brain damage has been addressed using global knockout mice for Px1 and pannexin-2 (Px2), two pannexin proteins expressed in the nervous system. Contrasting with the deleterious effects of global Cx32 deficiency and astrocytic Cx43 deficiency, global combined deficiency of Px1 and $\mathrm{Px} 2$ results in protection against ischemic damage when evaluated $48 \mathrm{~h}$ after permanent middle cerebral artery occlusion (Bargiotas et al., 2011, 2012). Interestingly, astrocytes in primary culture with combined Px1 and Px2 deficiency show normal ATP release and membrane conductance, indicating that pannexins do not contribute significantly to astrocytic channel function in these in vitro conditions. In contrast, Px1- and Px2-deficient cortical neurons in primary culture show defective dye release (Bargiotas et al., 2011). These results suggest that pannexins contribute to ischemic damage expansion through a neurondependent mechanism. This is supported by a study showing comparable degrees of protection against ischemia-reperfusion damage in retinas of global and neuron-selective Px1 deficiency (Dvoriantchikova et al., 2012). Interestingly, microglial cells of Px1 deficient mice retain their ability to display rapid morphologic plasticity in response to extracellular ATP and other stimuli associated with injury (Dissing-Olesen et al., 2014). However, a possible contribution of microglia or endothelial cells (Gaynullina et al., 2014; Sharma et al., 2018) to explain the neuroprotective effect of global Px1 and Px2 deletion cannot be ruled out and needs to be specifically tested.

The availability of new mouse lines to achieve conditional deletion of Px1 using Cre-lox technology (Dvoriantchikova et al., 2012) and conditional expression of tamoxifen-inducible Cre recombinase in microglia and leptomeninges, sparing blood monocytes (Kaiser and Feng, 2019) is much promising. Given that Px2 can compensate for Px1 deficiency, and vice versa (Bargiotas et al., 2011) an in vivo approach to address the impact of microglia-selective Px1 deficiency on ischemic damage and other pathologic conditions will likely need to combine the conditional microglia-selective Px1 deletion with a global (or conditional, when available) Px2 deletion. This is an analogous strategy to the one used to address the roles of astrocytic $\mathrm{Cx} 43$ without compensation by Cx30 (Wallraff et al., 2006). One additional factor that has to be taken into consideration is the observation of sexual dimorphism in the neuroprotective effect of global Px1 deficiency. When evaluated $48 \mathrm{~h}$ after middle cerebral artery occlusion without sex discrimination, global Pxl deficiency did not show protection against ischemic damage (Bargiotas et al., 2011). Of note, another study using a middle cerebral artery occlusion lesion model in an independently generated Px1-knockout line observed that females with Px1 deficiency showed approximately $50 \%$ smaller size infarcts 4 days after the lesion when compared with wild type females (FreitasAndrade et al., 2017). In contrast, males with Px1 deficiency displayed lesion sizes that were indistinguishable from lesions in wild type male mice. The differential susceptibility to ischemic damage between males and females correlated with differential astrocytic reactivity and degree of neuroinflamation, assessed through GFAP and Ibal immunostaining, respectively, which were less dramatic in Px1-deficient female mice when compared to wild type female mice and males of either genotype (Freitas-Andrade et al., 2017). These data highlight the need to analyze the possibility for sexually dimorphic responses to nervous system insults before concluding a lack of effect of manipulations.

Overall, the current available data strongly suggest that therapeutic interventions to prevent ischemic damage will benefit from strengthening the efforts to develop tools to counteract connexin and pannexin hemichannel activity while preserving connexin gap junction function.

\section{SPINAL CORD INJURY}

Female mice with selective astrocytic $\mathrm{Cx} 43$ deficiency in a Cx30-knockout background were also assessed to evaluate their response to spinal cord contusion injury, and compared to controls that had only the global Cx30 deficiency (Huang et al., 2012). This study reported that the response to weight-drop spinal cord injury involved less ATP release in the peritraumatic area $1 \mathrm{~h}$ after injury in mice with astrocytic $\mathrm{Cx} 43$ deficiency. These mice also showed reduced astrocytic and microglial reactivity 1 week after injury and smaller lesion sizes than control mice when evaluated 8 weeks after injury, accompanied by an improved functional recovery that was observed as early as 3 days after damage (Huang et al., 2012). Thus, in this injury model, $\mathrm{Cx} 43$ availability was deleterious. In another model of spinal cord contusion injury in rats, peptide5, a $\mathrm{Cx} 43$ mimetic peptide that blocks Cx43 hemichannels and gap junctions (O'Carroll et al., 2008), administered at the lesion site $1 \mathrm{~h}$ after the lesion, diminished astrocytic activation and inflammatory cell numbers at the lesion site, promoted neuronal survival, and improved locomotor recovery when compared with the administration of a control peptide (O'Carroll et al., 2013). These data are consistent with the more selective astrocyte-directed manipulation of $\mathrm{Cx} 43$ mentioned above and support the idea that $\mathrm{Cx} 43$ is deleterious for the outcome of traumatic spinal cord injury. Interestingly, comparable results were obtained when peptide5 was administered systemically to rats with spinal cord contusion (Mao et al., 2017b). Consistent results were obtained in yet another study using female mice treated systemically with INI-0602, a derivative of the broadly used non-selective connexin and pannexin blocker carbenoxolone (Bruzzone et al., 2005; Dahl et al., 2013), that prevents the release of glutamate from mouse cultured microglia in response to lipopolysaccharide administration (Takeuchi et al., 2011). Administration of INI-0602, starting at the time of injury and during a month, improved the outcome of transection spinal cord injury, reducing astrogliosis and inflammation, and improving motor recovery (Umebayashi et al., 2014). These results emphasize the idea that even when cell-type 
selective manipulations are essential to address the mechanisms behind the resolution of injuries, non-selective manipulations have potential as useful treatments, provided that there are no significant undesired off-targets for the therapeutic agent (Mao et al., 2017a).

In contrast to what was reported in spinal cord injury models, mice with astrocytic $\mathrm{Cx} 43$ deletion in a wild type Cx30 background increased the extent of astrogliosis and inflammatory cells around the lesioned area in a brain stab wound injury model (Theodoric et al., 2012). A number of factors may explain these differences, such as the type of injury, the lesion site, the time of observation after injury and the cellular microenvironment around the wound.

\section{NEUROPATHIC PAIN}

The development of neuropathic pain after peripheral or central damage involves plastic changes in spinal nociceptive circuits that involve glial plasticity as well. Selective manipulation of astrocytes and microglia have recently revealed that connexin and pannexin proteins in these glial types are critical for the development of neuropathic pain. In a mouse model of mild spinal cord injury, selective astrocytic Cx43 deficiency in a Cx30 knockout background reduced the development of mechanical allodynia and heat hyperalgesia, when compared with $\mathrm{Cx} 30$ knockout and wild type mice (Chen et al., 2012). On the other hand, Pxl deficiency in myeloid cells including microglia attenuated the development of mechanical allodynia in a model of induced osteoarthritis by intra-articular administration of monosodium iodoacetate. Interestingly, the hyperactivity of spinal dorsal horn neurons associated with the development of mechanical allodynia was also attenuated by myeloid cell-selective deletion of Px1 (Mousseau et al., 2018). It is important to note that this study used an inducible knock-in/knock-out Cre-lox system that allowed the researchers to evaluate the acute effects of eliminating Px1 expression in the myeloid lineage. Another study used a model in which mechanical allodynia also develops after spared nerve injury of the sciatic nerve. This study did not observe a reduction of pain thresholds in mice with Px1 deficiency in the myeloid lineage using a non-inducible transgenic Cre-lox system under comparable regulatory sequences (the $\mathrm{Cx} 3 \mathrm{crl}$ promoter; Weaver et al., 2017). In contrast, this same study did observe a prevention of nerve injury-induced mechanical allodynia in global Px1-deficient mice. These results suggest that the non-inducible deletion of Px1 from myeloid cells can be developmentally compensated, while the global Px1 deletion cannot. Strikingly, Weaver et al. could reinstate the capability for nerve injury-induced mechanical allodynia in global Px1-deficient mice after wild type bone marrow transplantation, indicating that bone marrow-derived immune cells confer at least part of the Px1 dependence of nerve injury-induced mechanical allodynia. Combined manipulations of astrocytic connexins and microglial pannexins in specific time windows will help elucidate whether and how these cell types and molecules interact for the development of neuropathic pain. The availability of tools to genetically manipulate astrocytes and microglia with different inductor drugs (Tanaka et al., 2012; Srinivasan et al., 2016) is a promising new avenue with potential to assess interactions between these cell types. The generation of new tools designed to achieve targeted deletions of a broader diversity of connexins and pannexins than what is available (Liao et al., 2001; Cohen-Salmon et al., 2002; Poon et al., 2014; Clasadonte et al., 2017) will overcome one of the current limitations.

\section{TUMORS}

Besides their participation in the resolution of nervous system damage, astrocytes are determinants of the microenvironment in which brain tumors develop. In addition, tumor cells express connexins and there is evidence using dye transfer in vitro and in vivo that they can establish connexin-mediated gap junctions with non-tumor cells (Zhang et al., 1999, 2003). However, a direct electrophysiological measurement of gap junction coupling between astrocytes and tumor cells is still lacking. Data supporting the relevance of connexin proteins as potential targets for antitumor therapy have been reviewed recently (Aasen et al., 2017; Umans and Sontheimer, 2018; Uzu et al., 2018). Whether connexin gap junction, hemichannel and/or non-channel functions are important for tumor progression has not been resolved. However, there is compelling evidence that some tumor cells downregulate connexin proteins and that restoring connexin expression reduces malignancy. As an example, C6 rat glioma cells express low levels of $\mathrm{Cx} 43$ and $\mathrm{Cx} 30$. When implanted intracranially, they produce well-defined large tumors in the brain parenchyma, resulting in a survival rate of $43 \%$ of transplanted individuals after 30 days of implantation. However, rats implanted with Cx30 cDNA-transfected C6 glioma cells exhibited $66 \%$ of survival rate, and smaller tumor masses were detected by MRI analysis (Arun et al., 2017). Interestingly, Px1-transfected C6 glioma cells in culture showed reduced proliferation and motility and displayed dye coupling, which was virtually absent in untransfected C6 cells (Lai et al., 2007). When inoculated to nude mice, Px1-transfected C6 glioma cells produced significantly smaller tumors than cells transfected with a control construct. Similar results were observed for Px2-transfected C6 glioma cells (Lai et al., 2009).

Contrasting with the former results, in a brain metastasis model using nude mice inoculated with mammary adenocarcinoma cells of human origin via the left cardiac ventricle, shRNA-mediated depletion of $\mathrm{Cx} 43$ from cancerous cells reduced metastatic growth in the brain parenchyma when assessed 2 weeks after inoculation (Chen et al., 2016, 2017). Wild-type Cx43 re-expression in Cx43-depleted cancerous cells rescued metastatic activity, whereas re-expression of a mutated variant of $\mathrm{Cx} 43$ that does not form functional channels [Cx43(T154A)] did not (Beahm et al., 2006). Interestingly, $\mathrm{Cx} 43$ promoted proliferation of metastatic cells in the brain, but not extravasation across the bloodbrain barrier, as $\mathrm{Cx} 43$ depletion in cancer cells did not significantly diminish the number of cancer cells in the 


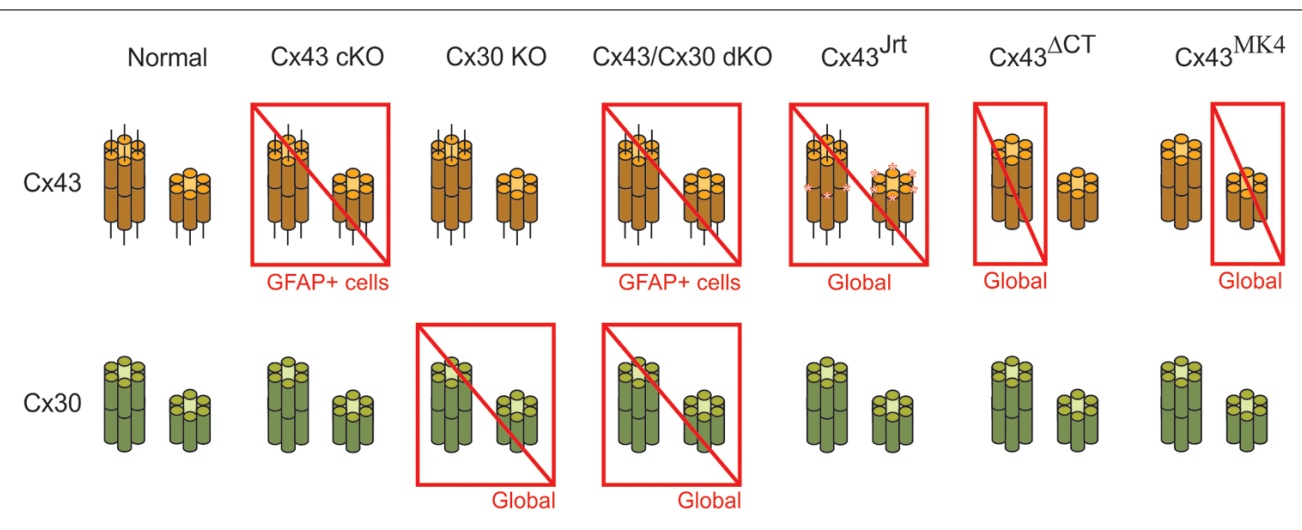

Toxic amyloid exposure Dye uptake

Neuronal death
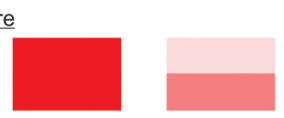

Orellana et al., 2011
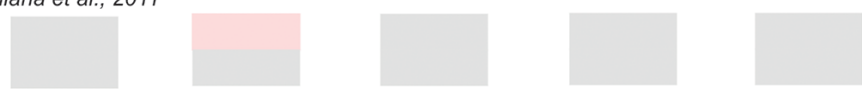

Experimental autoimmune encephalomyelitis

Early motor signs

Early microgliosis

Late motor signs

Late microgliosis

Late astrogliosis
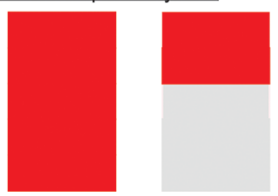

Lutz et al., 2012, Fang et al, 2018

APP/PS1 mice

Dye uptake

Neuronal damage

Astrogliosis

Congnitive impair
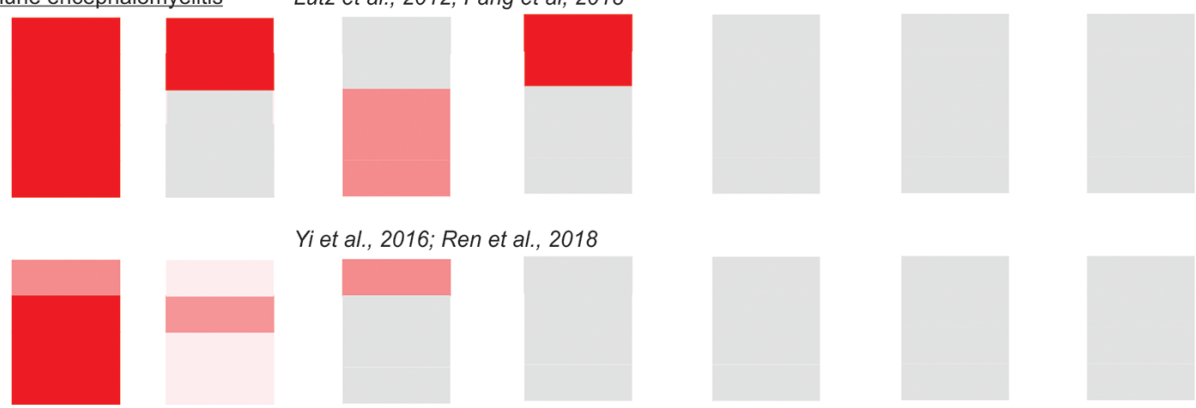

Ischemia

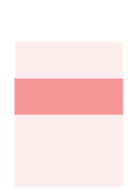

Yi et al., 2016; Ren et al., 2018

Infarct size

Dye uptake
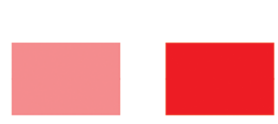

Nakase et al., 2003; Lin et al., 2008; Kozoriz et al., 2013; Freitas-Andrade et al, 2019

Spinal cord injury

ATP release

Astrogliosis

Motor impair
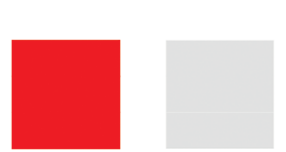

Huang et al., 2012
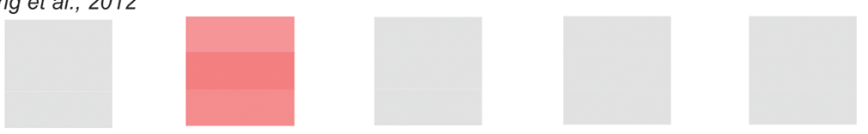

Neuropathic pain

Astrogliosis

Allodynia/hyperalgesia
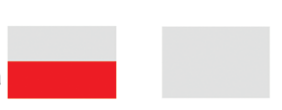

Chen et al., 2012

Stab wound

Astrogliosis

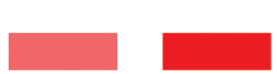

Theodoric et al., 2012

Glioma

Astrogliosis

Tumor infiltration
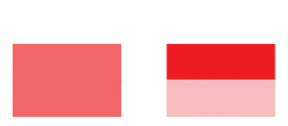

Sin et al., 2016
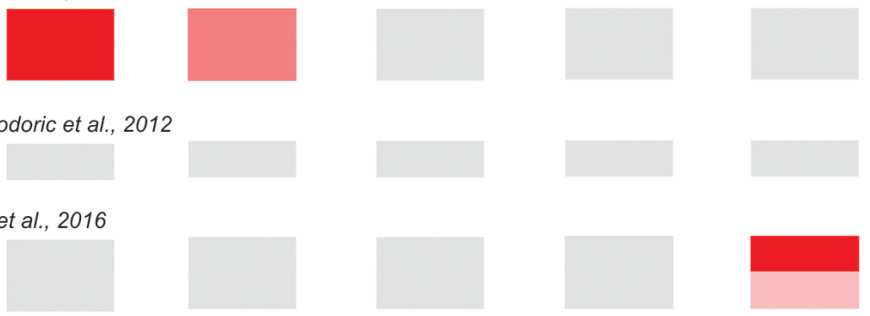

No data

Severity

FIGURE 1 | Summary of the impact of global and astrocyte-selective connexin function disruption in the context of in vivo models of central nervous system disease.

brain parenchyma, but micrometastases showed decreased proliferative activity when assessed 1 week after cancer cell inoculation. Based on a large body of accompanying in vitro data, the authors proposed a model in which gap junctions between tumor cells and astrocytes are required for cancer cell growth and survival (Chen et al., 2016). However, a potential hemichannel function of $\mathrm{Cx} 43$ in this process cannot be discarded. 


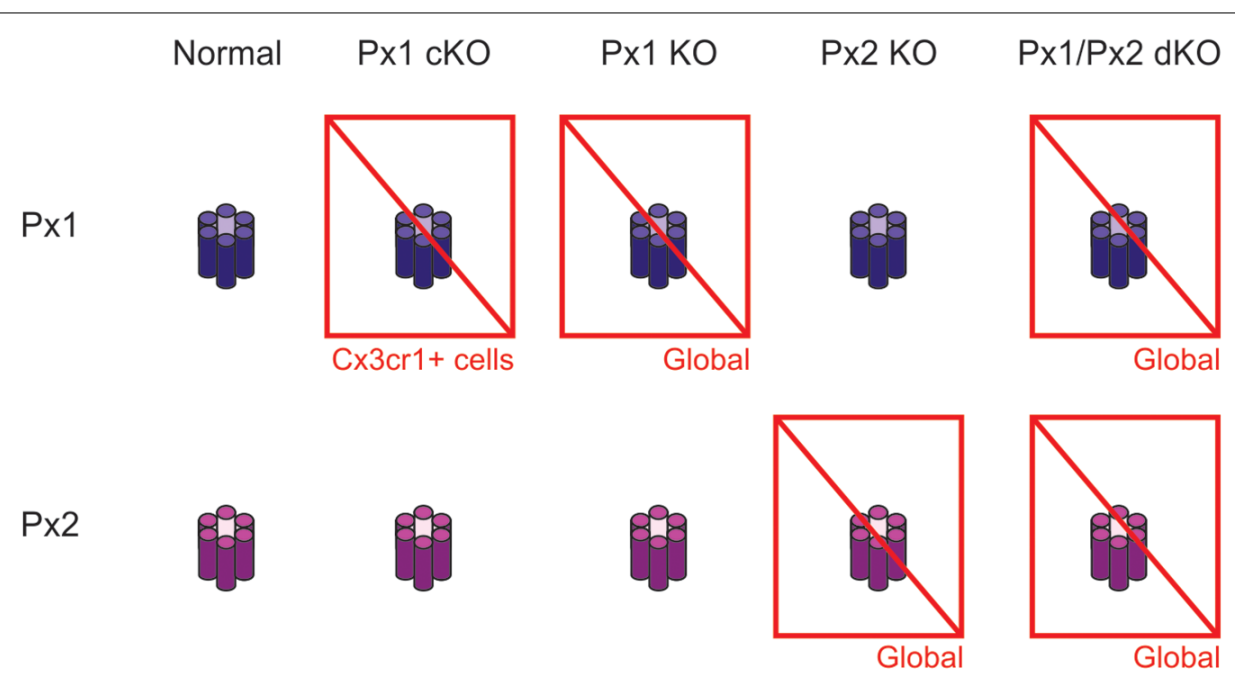

\section{Experimental auto \\ Early microgliosis \\ Late motor signs \\ Late microgliosis \\ Late astrogliosis \\ ATP release}

Ischemia

Infarct size

Neuropathic pain

Allodynia/hyperalgesia

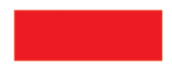

Glioma

Tumor proliferation
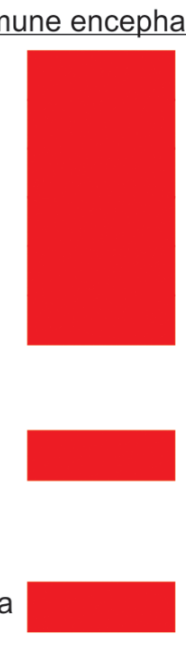

myelitis

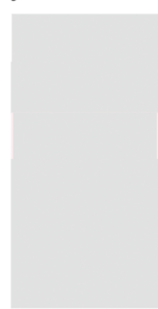

Bargiotas et at, 2011
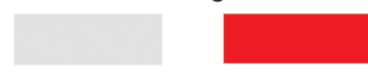

Weaver et al., 2017; Mousseau et al, 2018

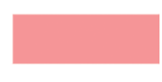

Lai et al., 2007, 2009

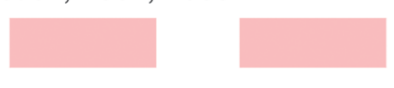

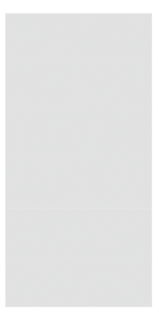
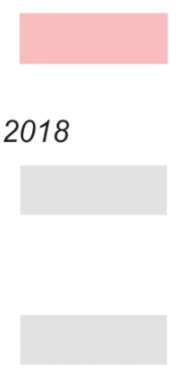

\section{No data}

Severity

FIGURE 2 | Summary of the impact of global and myeloid cell-selective pannexin function disruption in the context of in vivo models of central nervous system disease.

Further research with in vivo models and tumor cell manipulations targeting specific connexin functions will shed light on the roles of connexins expressed by cancer cells for their capacity to invade the brain parenchyma, their proliferation and survival, which may differ depending on cancer cell type. To conclude, we will focus on the analysis of plasticity of astrocyte connexin functions in the proximity of tumor cells and their possible detrimental or beneficial effects for tumor progression.
In mice with $\mathrm{Cx} 43$-deficient astrocytes, brain implantation of mouse GL261 glioma cells resulted in a larger extent of astrogliosis near the tumor implant, together with reduced invasion of tumor cells into the brain parenchyma. This result was replicated when Cx43-knockdown glioma cells were implanted in wild type mice (Sin et al., 2016). To determine which $\mathrm{Cx} 43$ function was taking part in this process, glioma cells were transfected with cDNA coding for the channel-defective Cx43 mutant variant, Cx43(T154A), 
TABLE 2 | Genetic tools of potential application to achieve inducible connexin and pannexin manipulations of glial cells in vivo.

\begin{tabular}{|c|c|c|c|c|}
\hline \multicolumn{5}{|c|}{ Cre-expressing lines } \\
\hline $\begin{array}{l}\text { Cell-type selectivity } \\
\text { (in nervous system) }\end{array}$ & Construct & Induction method & RRID & Reference \\
\hline Astrocytes & $\begin{array}{l}\text { Aldh1l1-Cre/ERT2 BAC } \\
\text { transgene }\end{array}$ & Tamoxifen & IMSR_JAX:029655 & Srinivasan et al. (2016) \\
\hline $\begin{array}{l}\text { Astrocytes, adult neural } \\
\text { progenitors cells }\end{array}$ & Nestin-CreER transgene & Tamoxifen & IMSR_JAX:012906 & Burns et al. (2007) \\
\hline $\begin{array}{l}\text { Astrocytes, adult neural } \\
\text { progenitors cells }\end{array}$ & $\begin{array}{l}\text { PAC } \\
\text { Fgfr3-icreERT2 transgene }\end{array}$ & Tamoxifen & IMSR_JAX:025809 & Rivers et al. (2008) \\
\hline $\begin{array}{l}\text { Oligodendrocytes, } \\
\text { Schwann cells, olfactory } \\
\text { ensheathing glia, adult } \\
\text { neural progenitors }\end{array}$ & PLP1-Cre/ERT transgene & Tamoxifen & IMSR_JAX:005975 & $\begin{array}{l}\text { Doerflinger et al. (2003), } \\
\text { Guo et al. (2010) and } \\
\text { Piantanida et al. (2019) }\end{array}$ \\
\hline $\begin{array}{l}\text { NG2 cells (oligodendrocyte } \\
\text { and astrocyte precursors) }\end{array}$ & $\begin{array}{l}\text { NG2-Cre/ER }{ }^{T M} \text { BAC } \\
\text { transgene }\end{array}$ & Tamoxifen & IMSR_JAX:008538 & Zhu et al. (2011) \\
\hline $\begin{array}{l}\text { Microglia, mononuclear } \\
\text { phagocyte system }\end{array}$ & Cx3cr1-Cre/ER transgene & Tamoxifen & IMSR_JAX:020940 & $\begin{array}{l}\text { Yona et al. (2013) and Zhao } \\
\text { et al. (2019) }\end{array}$ \\
\hline $\begin{array}{l}\text { Microglia, mononuclear } \\
\text { phagocyte system }\end{array}$ & $\begin{array}{l}\text { Cx3cr1-Cre/ER/YFP } \\
\text { transgene }\end{array}$ & Tamoxifen & IMSR_JAX:021160 & Parkhurst et al. (2013) \\
\hline $\begin{array}{l}\text { Microglia, mononuclear } \\
\text { phagocyte system }\end{array}$ & lba1-tTA transgene & Doxicycline & IMSR_RBRC05769 & Tanaka et al. (2012) \\
\hline \multicolumn{5}{|c|}{ Cre-responding lines } \\
\hline $\begin{array}{l}\text { Cell-type selectivity (in } \\
\text { nervous system) }\end{array}$ & Construct & $\begin{array}{l}\text { Expressed } \\
\text { protein/s }\end{array}$ & RRID & Reference \\
\hline Astrocytes & $\begin{array}{l}\text { Aldh1l1-loxP-EGFP- } \\
\text { 4XpolyA-loxP-diptheria } \\
\text { toxin A (DTA)-polyA BAC } \\
\text { transgene }\end{array}$ & $\begin{array}{l}\text { EGFP before } \\
\text { recombination, DTA } \\
\text { after recombination }\end{array}$ & IMSR_JAX:026033 & Tsai et al. (2012) \\
\hline \multicolumn{5}{|c|}{ Viral vectors } \\
\hline $\begin{array}{l}\text { Cell-type selectivity (in } \\
\text { nervous system) }\end{array}$ & Construct & $\begin{array}{l}\text { Expressed } \\
\text { protein/s }\end{array}$ & RRID & Reference \\
\hline $\begin{array}{l}\text { Astrocytes (adult } \\
\text { non-neurogenic regions) }\end{array}$ & $\operatorname{AAV}(5)-G F A P(2.2)-i C r e$ & Cre recombinase & & García-Cáceres et al. (2016) \\
\hline Astrocytes & AAV-DJ-hALDH1L1-Cre & Cre recombinase & & Koh et al. (2017) \\
\hline \multicolumn{5}{|c|}{ Connexin and pannexin floxed lines } \\
\hline Targeted gene & Targeted sequence & $\begin{array}{l}\text { Targeted } \\
\text { connexins }\end{array}$ & RRID & Reference \\
\hline Gja1 & Exon 2 & $\mathrm{C} \times 43$ & IMSR_JAX:008039 & $\begin{array}{l}\text { Liao et al. (2001) and } \\
\text { Clasadonte et al. (2017) }\end{array}$ \\
\hline Gjb2 & Exon 2 & $\mathrm{C} \times 26$ & & Cohen-Salmon et al. (2002) \\
\hline Panx1 & Exon 3/4 & Px1 & & $\begin{array}{l}\text { Dvoriantchikova et al. } \\
\text { (2012) and Poon et al. } \\
\text { (2014) }\end{array}$ \\
\hline
\end{tabular}

and implanted in astrocyte-selective Cx43-deficient mice, or control mice. Tumor invasion was compromised only in the astrocyte $\mathrm{Cx} 43$ null background, suggesting that heterocellular (glioma-astrocyte) gap junction channel activity is not necessary for tumor invasion. However, release of astrocyte gliotransmitters via $\mathrm{Cx} 43$ hemichannels cannot be discarded. In addition, the outcome of implanted glioma cells was assessed in $\mathrm{Cx} 43^{\Delta \mathrm{CT} /-}$ mice carrying one astrocyte-selective null $\mathrm{Cx} 43$ allele and one $\triangle \mathrm{CT}$ allele that consists in a truncated $\mathrm{Cx} 43$ variant (Cx43K258stop). This variant lacks a cytoplasmic tail that carries phosphorylation sites and protein-interacting domains (Freitas-Andrade et al., 2019) and exhibits reduced gap junction activity and increased hemichannel activity when assessed in cultured astrocytes using dye transfer and uptake techniques (Sin et al., 2016). Cx43 $4 \mathrm{CT} /-$ mice displayed compromised invasion of tumor cells in the brain parenchyma and increased astrogliosis when compared with $\mathrm{Cx} 43^{+/-}$(mice carrying one astrocyte-selective Cx43 null allele and one wild type allele; Sin et al., 2016). These results indicate that astrocytic Cx43 constitutes a means for tumor invasion in the brain. The channel and non-channel functions directly involved in tumor invasion and survival are still elusive; however, at least in the former case, the results seem to discard adhesive 
properties of the extracellular loops, which remained intact in the mutant variants used, provided that $\mathrm{Cx} 43$ expression levels and traffic to the membrane remained invariant.

\section{CONCLUDING REMARKS AND FUTURE DIRECTIONS}

A summary of the cases of connexin- and pannexin-deficient mouse models reviewed is shown in Figures 1, 2. One interesting feature to highlight is that astrogliosis appears dissociated from the availability of functional astrocytic connexins and is likely more related with the inflammatory environment associated with each particular case. The data reviewed gives support to the idea that the hyperactivation of glial hemichannels leads to circuit dysfunction and worsens neurodegeneration in a variety of contexts. The generally beneficial effect of pannexin deficiency vs. the more variable outcomes observed for connexin deficiency supports the idea that connexin proteins can form both hemichannels and gap junctions, while pannexin proteins are predominantly identified with hemichannel function. Efforts to develop blood-brain barrier-permeant connexin hemichannel blockers for systemic administration appear as promising therapeutic tools. Tools to favor the bias of connexins towards gap junction assembly at the expense of hemichannel availability are expected to be beneficial.

Future studies exploiting the advantages of cell-type specific connexin and/or pannexin disruption, time control provided by inducible genetic drivers or vector administration (Table 2) and dissociated manipulation of gap junction channel, hemichannel and non-channel functions will contribute to address the roles of connexins in different nervous system pathologies to better design adequate therapeutic interventions. The focus on cell types whose roles are only beginning to be explored as participants in neuroinflammation (Table 1) is a fertile field for new research. One interesting case is NG2 glia, known to be precursors of mature oligondendrocytes present in numbers comparable to astrocytes in many brain areas (Nishiyama et al., 2005) and to be components of the glial scar after traumatic injury (Schäfer and Tegeder, 2018). Of particular importance is the

\section{REFERENCES}

Aasen, T., Mesnil, M., Naus, C. C., Lampe, P. D., and Laird, D. W. (2017). Gap junctions and cancer: communicating for 50 years. Nat. Rev. Cancer 17:74. doi: $10.1038 /$ nrc.2016.142

Abudara, V., Retamal, M. A., Del Rio, R., and Orellana, J. A. (2018). Synaptic functions of hemichannels and pannexons: a double-edged sword. Front. Mol. Neurosci. 11:435. doi: 10.3389/fnmol.2018.00435

Arun, S., Ravisankar, S., and Vanisree, A. J. (2017). Implication of connexin30 on the stemness of glioma: connexin30 reverses the malignant phenotype of glioma by modulating IGF-1R, CD133 and cMyc. J. Neurooncol. 135, 473-485. doi: 10.1007/s11060-017-2608-4

Bargiotas, P., Krenz, A., Hormuzdi, S. G., Ridder, D. A., Herb, A., Barakat, W., et al. (2011). Pannexins in ischemia-induced neurodegeneration. Proc. Natl. Acad. Sci. U S A 108, 20772-20777. doi: 10.1073/pnas.1018262108 availability of genetic tools to manipulate NG2 glia selectively (Zhu et al., 2011).

In addition, forthcoming studies will need to address complex interactions arising from combined selective connexin deficiency in more than one cell type. We should not be surprised by finding regional, age and pathology-dependent differences instead of a more general rule. Figures 1, 2 highlight the complexity of drawing unequivocal conclusions regarding the relevance of hemichannel, gap junction and non-channel functions of connexins and pannexins. Despite the availability of a substantial diversity of genetic tools to distinguish among these functions, it is uncommon to find comparisons of their effects in the same disease model with experiments run in parallel to minimize factors of variability. We envision that collaborative work of different groups with the aim of shedding light on this question will generate invaluable new and confirmative data crucial for the design of new therapies.

\section{AUTHOR CONTRIBUTIONS}

LB and LR wrote the manuscript. LA and AP revised the manuscript and made important editions.

\section{FUNDING}

This work was supported by Secretaría de Ciencia, Tecnología e Innovación Productiva, Fondo para la Investigación Científica y Tecnológica (PICT 2014-1954, Argentina), that provided funding for research cited in this review, stipend for LA and access to subscription-based journals, Universidad de Buenos Aires (136901), that provided infrastructure and salary for LR and Consejo Nacional de Investigaciones Científicas y Técnicas (142692), that provided salary for LR, AP and LB.

\section{ACKNOWLEDGMENTS}

We wish to thank the members of the Glia Club at the School of Medicine, University of Buenos Aires, for providing an encouraging environment for critical discussion about glia functions.

Bargiotas, P., Krenz, A., Monyer, H., and Schwaninger, M. (2012). Functional outcome of pannexin-deficient mice after cerebral ischemia. Channels 6, 453-456. doi: 10.4161/chan.22315

Beahm, D. L., Oshima, A., Gaietta, G. M., Hand, G. M., Smock, A. E., Zucker, S. N., et al. (2006). Mutation of a conserved threonine in the third transmembrane helix of $\alpha$ - and $\beta$-connexins creates a dominant-negative closed gap junction channel. J. Biol. Chem. 281, 7994-8009. doi: 10.1074/jbc.m506533200

Belousov, A. B., Fontes, J. D., Freitas-Andrade, M., and Naus, C. C. (2017). Gap junctions and hemichannels: communicating cell death in neurodevelopment and disease. BMC Cell Biol. 18:4. doi: 10.1186/s12860-016-0120-x

Bosch, M., and Kielian, T. (2014). Hemichannels in neurodegenerative diseases: is there a link to pathology? Front. Cell. Neurosci. 8:242. doi: 10.3389/fncel.2014. 00242

Bruzzone, R., Barbe, M. T., Jakob, N. J., and Monyer, H. (2005). Pharmacological properties of homomeric and heteromeric pannexin hemichannels expressed in 
Xenopus oocytes. J. Neurochem. 92, 1033-1043. doi: 10.1111/j.1471-4159.2004. 02947.x

Burns, K. A., Ayoub, A. E., Breunig, J. J., Adhami, F., Weng, W. L., Colbert, M. C., et al. (2007). Nestin-CreER mice reveal DNA synthesis by nonapoptotic neurons following cerebral ischemia hypoxia. Cereb. Cortex 17, 2585-2592. doi: $10.1093 /$ cercor/bhl164

Chen, Q., Boire, A., Jin, X., Valiente, M., Er, E. E., Lopez-Soto, A., et al. (2016). Carcinoma-astrocyte gap junctions promote brain metastasis by cGAMP transfer. Nature 533, 493-498. doi: 10.1038/nature18268

Chen, Q., Boire, A., Jin, X., Valiente, M., Emrah Er, E., Lopez-Soto, A., et al. (2017). Corrigendum: carcinoma-astrocyte gap junctions promote brain metastasis by cGAMP transfer. Nature 544:124. doi: 10.1038/nature21730

Chen, L., and Brosnan, C. F. (2006). Exacerbation of experimental autoimmune encephalomyelitis in $\mathrm{P} 2 \mathrm{X}_{7} \mathrm{R}^{-/}$mice: evidence for loss of apoptotic activity in lymphocytes. J. Immunol. 176, 3115-3126. doi: 10.4049/jimmunol.176.5.3115

Chen, M. J., Kress, B., Han, X., Moll, K., Peng, W., Ji, R. R., et al. (2012). Astrocytic CX43 hemichannels and gap junctions play a crucial role in development of chronic neuropathic pain following spinal cord injury. Glia 60, 1660-1670. doi: $10.1002 /$ glia. 22384

Chen, B., Yang, L., Chen, J., Chen, Y., Zhang, L., Wang, L., et al. (2019). Inhibition of Connexin43 hemichannels with Gap19 protects cerebral ischemia/reperfusion injury via the JAK2/STAT3 pathway in mice. Brain Res. Bull. 146, 124-135. doi: 10.1016/j.brainresbull.2018.12.009

Clasadonte, J., Scemes, E., Wang, Z., Boison, D., and Haydon, P. G. (2017). Connexin 43-mediated astroglial metabolic networks contribute to the regulation of the sleep-wake cycle. Neuron 95, 1365.e5-1380.e5. doi: 10.1016/j. neuron.2017.08.022

Cohen-Salmon, M., Ott, T., Michel, V., Hardelin, J. P., Perfettini, I., Eybalin, M., et al. (2002). Targeted ablation of connexin26 in the inner ear epithelial gap junction network causes hearing impairment and cell death. Curr. Biol. 12, 1106-1111. doi: 10.1016/s0960-9822(02)00904-1

Contreras, J. E., Sánchez, H. A., Véliz, L. P., Bukauskas, F. F., Bennett, M. V., and Sáez, J. C. (2004). Role of connexin-based gap junction channels and hemichannels in ischemia-induced cell death in nervous tissue. Brain Res. Rev. 47, 290-303. doi: 10.1016/j.brainresrev.2004.08.002

Dahl, G., Qiu, F., and Wang, J. (2013). The bizarre pharmacology of the ATP release channel pannexin1. Neuropharmacology 75, 583-593. doi: 10.1016/j. neuropharm.2013.02.019

De Bock, M., Leybaert, L., and Giaume, C. (2017). Connexin channels at the glio-vascular interface: gatekeepers of the brain. Neurochem. Res. 42, 2519-2536. doi: 10.1007/s11064-017-2313-x

Dissing-Olesen, L., LeDue, J. M., Rungta, R. L., Hefendehl, J. K., Choi, H. B., and MacVicar, B. A. (2014). Activation of neuronal NMDA receptors triggers transient ATP-mediated microglial process outgrowth. J. Neurosci. 34, 10511-10527. doi: 10.1523/JNEUROSCI.0405-14.2014

Doerflinger, N. H., Macklin, W. B., and Popko, B. (2003). Inducible site-specific recombination in myelinating cells. Genesis 35, 63-72. doi: 10.1002/gene. 10154

Dvoriantchikova, G., Ivanov, D., Barakat, D., Grinberg, A., Wen, R., Slepak, V. Z., et al. (2012). Genetic ablation of Pannexin 1 protects retinal neurons from ischemic injury. PLoS One 7:e31991. doi: 10.1371/journal.pone.0031991

Eugenin, E. A., Basilio, D., Sáez, J. C., Orellana, J. A., Raine, C. S., Bukauskas, F., et al. (2012). The role of gap junction channels during physiologic and pathologic conditions of the human central nervous system. J. Neuroimmune Pharmacol. 7, 499-518. doi: 10.1007/s11481-012-9352-5

Fang, M., Yamasaki, R., Li, G., Masaki, K., Yamaguchi, H., Fujita, A., et al. (2018). Connexin 30 deficiency attenuates chronic but not acute phases of experimental autoimmune encephalomyelitis through induction of neuroprotective microglia. Front. Immunol. 9:2588. doi: 10.3389/fimmu.2018. 02588

Farahani, R., Pina-Benabou, M. H., Kyrozis, A., Siddiq, A., Barradas, P. C., Chiu, F. C., et al. (2005). Alterations in metabolism and gap junction expression may determine the role of astrocytes as "good samaritans" or executioners. Glia 50, 351-361. doi: 10.1002/glia.20213

Freitas-Andrade, M., Bechberger, J. F., MacVicar, B. A., Viau, V., and Naus, C. C. (2017). Pannexin1 knockout and blockade reduces ischemic stroke injury in female, but not in male mice. Oncotarget 8, 36973-36983. doi: 10.18632/oncotarget.16937
Freitas-Andrade, M., and Naus, C. C. (2016). Astrocytes in neuroprotection and neurodegeneration: the role of connexin 43 and pannexin1. Neuroscience 323 , 207-221. doi: 10.1016/j.neuroscience.2015.04.035

Freitas-Andrade, M., Wang, N., Bechberger, J. F., De Bock, M., Lampe, P. D., Leybaert, L., et al. (2019). Targeting MAPK phosphorylation of Connexin43 provides neuroprotection in stroke. J. Exp. Med. 216, 916-935. doi: $10.1084 /$ jem. 20171452

Gajardo-Gómez, R., Labra, V. C., and Orellana, J. A. (2016). Connexins and pannexins: new insights into microglial functions and dysfunctions. Front. Mol. Neurosci. 9:86. doi: 10.3389/fnmol.2016.00086

García-Cáceres, C., Quarta, C., Varela, L., Gao, Y., Gruber, T., Legutko, B., et al. (2016). Astrocytic insulin signaling couples brain glucose uptake with nutrient availability. Cell 166, 867-880. doi: 10.1016/j.cell.2016.07.028

Gaynullina, D., Tarasova, O. S., Kiryukhina, O. O., Shestopalov, V. I., and Panchin, Y. (2014). Endothelial function is impaired in conduit arteries of pannexin1 knockout mice. Biol. Direct. 9:8. doi: 10.1186/1745-6150-9-8

Giaume, C., and Theis, M. (2010). Pharmacological and genetic approaches to study connexin-mediated channels in glial cells of the central nervous system. Brain Res. Rev. 63, 160-176. doi: 10.1016/j.brainresrev.2009.11.005

Giaume, C., Kirchhoff, F., Matute, C., Reichenbach, A., and Verkhratsky, A. (2007). Glia: the fulcrum of brain diseases. Cell Death Differ. 14, 1324-1335. doi: $10.1038 /$ sj.cdd.4402144

Guo, F., Maeda, Y., Ma, J., Xu, J., Horiuchi, M., Miers, L., et al. (2010). Pyramidal neurons are generated from oligodendroglial progenitor cells in adult piriform cortex. J. Neurosci. 30, 12036-12049. doi: 10.1523/JNEUROSCI.1360-10.2010

Hahn, A. F., Ainsworth, P. J., Naus, C. C., Mao, J., and Bolton, C. F. (2000). Clinical and pathological observations in men lacking the gap junction protein connexin 32. Muscle Nerve Suppl. 9, S39-S48. doi: 10.1002/10974598(2000)999:9<::aid-mus8>3.3.co;2-3

Huang, C., Han, X., Li, X., Lam, E., Peng, W., Lou, N., et al. (2012). Critical role of connexin 43 in secondary expansion of traumatic spinal cord injury. J. Neurosci. 32, 3333-3338. doi: 10.1523/JNEUROSCI.1216-11.2012

Iglesias, R., Dahl, G., Qiu, F., Spray, D. C., and Scemes, E. (2009). Pannexin 1: the molecular substrate of astrocyte "hemichannels". J. Neurosci. 29, 7092-7097. doi: 10.1523/JNEUROSCI.6062-08.2009

Kaiser, T., and Feng, G. (2019). Tmem119-EGFP and Tmem119CreERT2 transgenic mice for labeling and manipulating microglia. eNeuro 6:ENEURO.0448-18.2019. doi: 10.1523/ENEURO.0448-18.2019

Karpuk, N., Burkovetskaya, M., Fritz, T., Angle, A., and Kielian, T. (2011). Neuroinflammation leads to region-dependent alterations in astrocyte gap junction communication and hemichannel activity. J. Neurosci. 31, 414-425. doi: 10.1523/JNEUROSCI.5247-10.2011

Kielian, T. (2008). Glial connexins and gap junctions in CNS inflammation and disease. J. Neurochem. 106, 1000-1016. doi: 10.1111/j.1471-4159.2008.05405.x

Koh, W., Park, Y. M., Lee, S. E., and Lee, C. J. (2017). AAV-mediated astrocyte-specific gene expression under human. Exp. Neurobiol. 26, 350-361. doi: 10.5607/en.2017.26.6.350

Köster-Patzlaff, C., Hosseini, S. M., and Reuss, B. (2007). Persistent Borna Disease Virus infection changes expression and function of astroglial gap junctions in vivo and in vitro. Brain Res. 1184, 316-332. doi: 10.1016/j.brainres.2007. 09.062

Koulakoff, A., Mei, X., Orellana, J. A., Sáez, J. C., and Giaume, C. (2012). Glial connexin expression and function in the context of Alzheimer's disease. Biochim. Biophys. Acta 1818, 2048-2057. doi: 10.1016/j.bbamem.2011.10.001

Kozoriz, M. G., Bechberger, J. F., Bechberger, G. R., Suen, M. W., Moreno, A. P., Maass, K., et al. (2010). The connexin43 C-terminal region mediates neuroprotection during stroke. J. Neuropathol. Exp. Neurol. 69, 196-206. doi: 10.1097/nen.0b013e3181cd44df

Kozoriz, M. G., Lai, S., Vega, J. L., Sáez, J. C., Sin, W. C., Bechberger, J. F., et al. (2013). Cerebral ischemic injury is enhanced in a model of oculodentodigital dysplasia. Neuropharmacology 75, 549-556. doi: 10.1016/j.neuropharm. 2013.05.003

Lai, C. P., Bechberger, J. F., and Naus, C. C. (2009). Pannexin2 as a novel growth regulator in C6 glioma cells. Oncogene 28, 4402-4408. doi: 10.1038/onc. 2009.283

Lai, C. P., Bechberger, J. F., Thompson, R. J., MacVicar, B. A., Bruzzone, R., and Naus, C. C. (2007). Tumor-suppressive effects of pannexin 1 in C6 glioma cells. Cancer Res. 67, 1545-1554. doi: 10.1158/0008-5472.can-06-1396 
Lapato, A. S., and Tiwari-Woodruff, S. K. (2018). Connexins and pannexins: at the junction of neuro-glial homeostasis and disease. J. Neurosci. Res. 96, 31-44. doi: $10.1002 /$ jnr. 24088

Leithe, E., Mesnil, M., and Aasen, T. (2018). The connexin 43 C-terminus: a tail of many tales. Biochim. Biophys. Acta Biomembr. 1860, 48-64. doi: 10.1016/j. bbamem.2017.05.008

Liao, Y., Day, K. H., Damon, D. N., and Duling, B. R. (2001). Endothelial cell-specific knockout of connexin 43 causes hypotension and bradycardia in mice. Proc. Natl. Acad. Sci. U S A 98, 9989-9994. doi: 10.1073/pnas.171305298

Lin, J. H., Lou, N., Kang, N., Takano, T., Hu, F., Han, X., et al. (2008). A central role of connexin 43 in hypoxic preconditioning. J. Neurosci. 28, 681-695. doi: 10.1523/JNEUROSCI.3827-07.2008

Liu, L., Su, Y., Yang, W., Xiao, M., Gao, J., and Hu, G. (2010). Disruption of neuronal-glial-vascular units in the hippocampus of ovariectomized mice injected with D-galactose. Neuroscience 169, 596-608. doi: 10.1016/j. neuroscience.2010.05.028

Lutz, S. E., González-Fernández, E., Ventura, J. C., Pérez-Samartín, A., Tarassishin, L., Negoro, H., et al. (2013). Contribution of pannexin1 to experimental autoimmune encephalomyelitis. PLoS One 8:e66657. doi: 10.1371/journal.pone.0066657

Lutz, S. E., Raine, C. S., and Brosnan, C. F. (2012). Loss of astrocyte connexins 43 and 30 does not significantly alter susceptibility or severity of acute experimental autoimmune encephalomyelitis in mice. J. Neuroimmunol. 245, 8-14. doi: 10.1016/j.jneuroim.2012.01.007

Mao, Y., Nguyen, T., Tonkin, R. S., Lees, J. G., Warren, C., O’Carroll, S. J., et al. (2017a). Characterisation of Peptide5 systemic administration for treating traumatic spinal cord injured rats. Exp. Brain Res. 235, 3033-3048. doi: 10.1007/s00221-017-5023-3

Mao, Y., Tonkin, R. S., Nguyen, T., O'Carroll, S. J., Nicholson, L. F., Green, C. R., et al. (2017b). Systemic administration of connexin43 mimetic peptide improves functional recovery after traumatic spinal cord injury in adult rats. J. Neurotrauma 34, 707-719. doi: 10.1089/neu.2016.4625

Mousseau, M., Burma, N. E., Lee, K. Y., Leduc-Pessah, H., Kwok, C. H. T., Reid, A. R., et al. (2018). Microglial pannexin-1 channel activation is a spinal determinant of joint pain. Sci. Adv. 4:eaas9846. doi: 10.1126/sciadv. aas 9846

Nakagawa, M., Takashima, H., Umehara, F., Arimura, K., Miyashita, F., Takenouchi, N., et al. (2001). Clinical phenotype in X-linked Charcot-MarieTooth disease with an entire deletion of the connexin 32 coding sequence. J. Neurol. Sci. 185, 31-37. doi: 10.1016/s0022-510x(01)00454-3

Nakase, T., Fushiki, S., Söhl, G., Theis, M., Willecke, K., and Naus, C. C. (2003). Neuroprotective role of astrocytic gap junctions in ischemic stroke. Cell Commun. Adhes. 10, 413-417. doi: 10.1080/714040461

Nakase, T., Söhl, G., Theis, M., Willecke, K., and Naus, C. C. (2004). Increased apoptosis and inflammation after focal brain ischemia in mice lacking connexin43 in astrocytes. Am. J. Pathol. 164, 2067-2075. doi: 10.1016/s00029440(10)63765-0

Nielsen, B. S., Hansen, D. B., Ransom, B. R., Nielsen, M. S., and MacAulay, N. (2017). Connexin hemichannels in astrocytes: an assessment of controversies regarding their functional characteristics. Neurochem. Res. 42, 2537-2550. doi: 10.1007/s11064-017-2243-7

Nishiyama, A., Yang, Z., and Butt, A. (2005). Astrocytes and NG2-glia: what's in a name? J. Anat. 207, 687-693. doi: 10.1111/j.1469-7580.2005.00489.x

O'Carroll, S. J., Alkadhi, M., Nicholson, L. F., and Green, C. R. (2008). Connexin 43 mimetic peptides reduce swelling, astrogliosis and neuronal cell death after spinal cord injury. Cell Commun. Adhes. 15, 27-42. doi: 10.1080/15419060802014164

O'Carroll, S. J., Gorrie, C. A., Velamoor, S., Green, C. R., and Nicholson, L. F. (2013). Connexin 43 mimetic peptide is neuroprotective and improves function following spinal cord injury. Neurosci. Res. 75, 256-267. doi: 10.1016/j.neures. 2013.01.004

Oguro, K., Jover, T., Tanaka, H., Lin, Y., Kojima, T., Oguro, N., et al. (2001). Global ischemia-induced increases in the gap junctional proteins connexin 32 (Cx32) and Cx36 in hippocampus and enhanced vulnerability of Cx32 knock-out mice. J. Neurosci. 21, 7534-7542. doi: 10.1523/jneurosci.21-19-07534.2001

Olympiou, M., Sargiannidou, I., Markoullis, K., Karaiskos, C., Kagiava, A., Kyriakoudi, S., et al. (2016). Systemic inflammation disrupts oligodendrocyte gap junctions and induces ER stress in a model of CNS manifestations of
X-linked Charcot-Marie-Tooth disease. Acta Neuropathol. Commun. 4:95. doi: 10.1186/s40478-016-0369-5

Orellana, J. A., Martinez, A. D., and Retamal, M. A. (2013). Gap junction channels and hemichannels in the CNS: regulation by signaling molecules. Neuropharmacology 75, 567-582. doi: 10.1016/j.neuropharm.2013.02.020

Orellana, J. A., Shoji, K. F., Abudara, V., Ezan, P., Amigou, E., Sáez, P. J., et al. (2011). Amyloid $\beta$-induced death in neurons involves glial and neuronal hemichannels. J. Neurosci. 31, 4962-4977. doi: 10.1523/JNEUROSCI.641710.2011

Orellana, J. A., Sáez, P. J., Shoji, K. F., Schalper, K. A., Palacios-Prado, N., Velarde, V., et al. (2009). Modulation of brain hemichannels and gap junction channels by pro-inflammatory agents and their possible role in neurodegeneration. Antioxid. Redox Signal. 11, 369-399. doi: 10.1089/ars. 2008.2130

Parkhurst, C. N., Yang, G., Ninan, I., Savas, J. N., Yates, J. R., Lafaille, J. J., et al. (2013). Microglia promote learning-dependent synapse formation through brain-derived neurotrophic factor. Cell 155, 1596-1609. doi: 10.1016/j.cell. 2013.11.030

Piantanida, A. P., Acosta, L. E., Brocardo, L., Capurro, C., Greer, C. A., and Rela, L. (2019). Selective Cre-mediated gene deletion identifies connexin 43 as the main connexin channel supporting olfactory ensheathing cell networks. J. Comp. Neurol. 527, 1278-1289. doi: 10.1002/cne.24628

Poon, I. K., Chiu, Y. H., Armstrong, A. J., Kinchen, J. M., Juncadella, I. J., Bayliss, D. A., et al. (2014). Unexpected link between an antibiotic, pannexin channels and apoptosis. Nature 507, 329-334. doi: 10.1038/nature13147

Quintanilla, R. A., Orellana, J. A., and von Bernhardi, R. (2012). Understanding risk factors for Alzheimer's disease: interplay of neuroinflammation, connexinbased communication and oxidative stress. Arch. Med. Res. 43, 632-644. doi: 10.1016/j.arcmed.2012.10.016

Rami, A., Volkmann, T., and Winckler, J. (2001). Effective reduction of neuronal death by inhibiting gap junctional intercellular communication in a rodent model of global transient cerebral ischemia. Exp. Neurol. 170, 297-304. doi: 10.1006/exnr.2001.7712

Rawanduzy, A., Hansen, A., Hansen, T. W., and Nedergaard, M. (1997). Effective reduction of infarct volume by gap junction blockade in a rodent model of stroke. J. Neurosurg. 87, 916-920. doi: 10.3171/jns.1997.87.6.0916

Ren, R., Zhang, L., and Wang, M. (2018). Specific deletion connexin43 in astrocyte ameliorates cognitive dysfunction in APP/PS1 mice. Life Sci. 208, 175-191. doi: 10.1016/j.lfs.2018.07.033

Retamal, M. A., Froger, N., Palacios-Prado, N., Ezan, P., Sáez, P. J., Sáez, J. C., et al. (2007). Cx43 hemichannels and gap junction channels in astrocytes are regulated oppositely by proinflammatory cytokines released from activated microglia. J. Neurosci. 27, 13781-13792. doi: 10.1523/jneurosci.2042-07.2007

Rivers, L. E., Young, K. M., Rizzi, M., Jamen, F., Psachoulia, K., Wade, A., et al. (2008). PDGFRA/NG2 glia generate myelinating oligodendrocytes and piriform projection neurons in adult mice. Nat. Neurosci. 11, 1392-1401. doi: $10.1038 / \mathrm{nn} .2220$

Rouach, N., Avignone, E., Même, W., Koulakoff, A., Venance, L., Blomstrand, F., et al. (2002). Gap junctions and connexin expression in the normal and pathological central nervous system. Biol. Cell 94, 457-475. doi: 10.1016/s02484900(02)00016-3

Sáez, P. J., Orellana, J. A., Vega-Riveros, N., Figueroa, V. A., Hernández, D. E., Castro, J. F., et al. (2013). Disruption in connexin-based communication is associated with intracellular $\mathrm{Ca}^{2+}$ signal alterations in astrocytes from Niemann-Pick type C mice. PLoS One 8:e71361. doi: 10.1371/journal.pone. 0071361

Schäfer, M. K. E., and Tegeder, I. (2018). NG2/CSPG4 and progranulin in the posttraumatic glial scar. Matrix Biol. 68-69, 571-588. doi: 10.1016/j.matbio. 2017.10.002

Sharma, A. K., Charles, E. J., Zhao, Y., Narahari, A. K., Baderdinni, P. K., Good, M. E., et al. (2018). Pannexin-1 channels on endothelial cells mediate vascular inflammation during lung ischemia-reperfusion injury. Am. J. Physiol. Lung Cell. Mol. Physiol. 315, L301-L312. doi: 10.1152/ajplung.00004.2018

Sin, W. C., Aftab, Q., Bechberger, J. F., Leung, J. H., Chen, H., and Naus, C. C. (2016). Astrocytes promote glioma invasion via the gap junction protein connexin43. Oncogene 35, 1504-1516. doi: 10.1038/onc.2015.210

Srinivasan, R., Lu, T. Y., Chai, H., Xu, J., Huang, B. S., Golshani, P., et al. (2016). New Transgenic mouse lines for selectively targeting astrocytes and studying 
calcium signals in astrocyte processes in situ and in vivo. Neuron 92, 1181-1195. doi: $10.1016 /$ j.neuron.2016.11.030

Takeuchi, H., and Suzumura, A. (2014). Gap junctions and hemichannels composed of connexins: potential therapeutic targets for neurodegenerative diseases. Front. Cell. Neurosci. 8:189. doi: 10.3389/fncel.2014.00189

Takeuchi, H., Mizoguchi, H., Doi, Y., Jin, S., Noda, M., Liang, J., et al. (2011). Blockade of gap junction hemichannel suppresses disease progression in mouse models of amyotrophic lateral sclerosis and Alzheimer's disease. PLoS One 6:e21108. doi: 10.1371/journal.pone.0021108

Tanaka, K. F., Matsui, K., Sasaki, T., Sano, H., Sugio, S., Fan, K., et al. (2012). Expanding the repertoire of optogenetically targeted cells with an enhanced gene expression system. Cell Rep. 2, 397-406. doi: 10.1016/j.celrep.2012.06.011

Theodoric, N., Bechberger, J. F., Naus, C. C., and Sin, W. C. (2012). Role of gap junction protein connexin 43 in astrogliosis induced by brain injury. PLoS One 7:e47311. doi: 10.1371/journal.pone.0047311

Theriault, E., Frankenstein, U. N., Hertzberg, E. L., and Nagy, J. I. (1997). Connexin43 and astrocytic gap junctions in the rat spinal cord after acute compression injury. J. Comp. Neurol. 382, 199-214. doi: 10.1002/(sici)10969861(19970602)382:2<199::aid-cne5>3.3.co;2-5

Tress, O., Maglione, M., Zlomuzica, A., May, D., Dicke, N., Degen, J., et al. (2011). Pathologic and phenotypic alterations in a mouse expressing a connexin47 missense mutation that causes Pelizaeus-Merzbacher-like disease in humans. PLoS Genet. 7:e1002146. doi: 10.1371/journal.pgen.1002146

Tsai, H. H., Li, H., Fuentealba, L. C., Molofsky, A. V., Taveira-Marques, R., Zhuang, H., et al. (2012). Regional astrocyte allocation regulates CNS synaptogenesis and repair. Science 337, 358-362. doi: 10.1126/science.1222381

Ul-Hussain, M., Olk, S., Schoenebeck, B., Wasielewski, B., Meier, C., Prochnow, N., et al. (2014). Internal ribosomal entry site (IRES) activity generates endogenous carboxyl-terminal domains of $\mathrm{Cx} 43$ and is responsive to hypoxic conditions. J. Biol. Chem. 289, 20979-20990. doi: 10.1074/jbc.m113. 540187

Umans, R. A., and Sontheimer, H. (2018). Combating malignant astrocytes: Strategies mitigating tumor invasion. Neurosci. Res. 126, 22-30. doi: 10.1016/j. neures.2017.09.010

Umebayashi, D., Natsume, A., Takeuchi, H., Hara, M., Nishimura, Y., Fukuyama, R., et al. (2014). Blockade of gap junction hemichannel protects secondary spinal cord injury from activated microglia-mediated glutamate exitoneurotoxicity. J. Neurotrauma 31, 1967-1974. doi: 10.1089/neu. 2013.3223

Uzu, M., Sin, W. C., Shimizu, A., and Sato, H. (2018). Conflicting roles of connexin 43 in tumor invasion and growth in the central nervous system. Int. J. Mol. Sci. 19:E1159. doi: 10.3390/ijms19041159

Wallraff, A., Kohling, R., Heinemann, U., Theis, M., Willecke, K., and Steinhauser, C. (2006). The impact of astrocytic gap junctional coupling on potassium buffering in the hippocampus. J. Neurosci. 26, 5438-5447. doi: 10.1523/jneurosci.0037-06.2006
Wang, N., De Vuyst, E., Ponsaerts, R., Boengler, K., Palacios-Prado, N., Wauman, J., et al. (2013). Selective inhibition of Cx43 hemichannels by Gap19 and its impact on myocardial ischemia/reperfusion injury. Basic Res. Cardiol. 108:309. doi: 10.1007/s00395-012-0309-x

Wang, Y., and Yin, F. (2016). A review of X-linked Charcot-Marie-Tooth disease. J. Child Neurol. 31, 761-772. doi: 10.1177/0883073815604227

Weaver, J. L., Arandjelovic, S., Brown, G., Mendu, S. K., Schappe, M. S., Buckley, M. W., et al. (2017). Hematopoietic pannexin 1 function is critical for neuropathic pain. Sci. Rep. 7:42550. doi: 10.1038/srep42550

Xing, L., Yang, T., Cui, S., and Chen, G. (2019). Connexin hemichannels in astrocytes: role in CNS disorders. Front. Mol. Neurosci. 12:23. doi: 10.3389/fnmol.2019.00023

Yi, C., Mei, X., Ezan, P., Mato, S., Matias, I., Giaume, C., et al. (2016). Astroglial connexin 43 contributes to neuronal suffering in a mouse model of Alzheimer's disease. Cell Death Differ. 23, 1691-1701. doi: 10.1038/cdd.2016.63

Yona, S., Kim, K. W., Wolf, Y., Mildner, A., Varol, D., Breker, M., et al. (2013). Fate mapping reveals origins and dynamics of monocytes and tissue macrophages under homeostasis. Immunity 38, 79-91. doi: 10.1016/j.immuni.2012.12.001

Zhang, W., Couldwell, W. T., Simard, M. F., Song, H., Lin, J. H., and Nedergaard, M. (1999). Direct gap junction communication between malignant glioma cells and astrocytes. Cancer Res. 59, 1994-2003.

Zhang, W., DeMattia, J. A., Song, H., and Couldwell, W. T. (2003). Communication between malignant glioma cells and vascular endothelial cells through gap junctions. J. Neurosurg. 98, 846-853. doi: 10.3171/jns.2003.98. 4.0846

Zhao, X. F., Alam, M. M., Liao, Y., Huang, T., Mathur, R., Zhu, X., et al. (2019). Targeting microglia using Cx3crl-Cre lines: revisiting the specificity. eNeuro 6:ENEURO.0114-19.2019. doi: 10.1523/eneuro.0114-19.2019

Zhou, J. Z., and Jiang, J. X. (2014). Gap junction and hemichannel-independent actions of connexins on cell and tissue functions-an update. FEBS Lett. 588, 1186-1192. doi: 10.1016/j.febslet.2014.01.001

Zhu, X., Hill, R. A., Dietrich, D., Komitova, M., Suzuki, R., and Nishiyama, A. (2011). Age-dependent fate and lineage restriction of single NG2 cells. Development 138, 745-753. doi: 10.1242/dev.047951

Conflict of Interest: The authors declare that the research was conducted in the absence of any commercial or financial relationships that could be construed as a potential conflict of interest.

Copyright $\odot 2019$ Brocardo, Acosta, Piantanida and Rela. This is an open-access article distributed under the terms of the Creative Commons Attribution License (CC BY). The use, distribution or reproduction in other forums is permitted, provided the original author(s) and the copyright owner(s) are credited and that the original publication in this journal is cited, in accordance with accepted academic practice. No use, distribution or reproduction is permitted which does not comply with these terms. 